\title{
Understanding surgical antimicrobial prescribing behaviour in the hospital setting: a systematic review and meta- ethnography protocol
}

\author{
Hazel Parker ${ }^{1 *}$ D, Julia Frost ${ }^{2}$, Nicky Britten ${ }^{2}$, Sophie Robinson ${ }^{2}$ and Karen Mattick²
}

\begin{abstract}
Background: Surgical specialities use extensive amounts of antimicrobials, and misuse has been widely reported, making them a key target for antimicrobial stewardship initiatives. Interventions informed by, and tailored to, a clear understanding of the contextual barriers to appropriate antimicrobial use are more likely to successfully improve practice. However, this approach has been under utilised. Our aim is to synthesise qualitative studies on surgical antimicrobial prescribing behaviour (APB) in hospital settings to explain how and why contextual factors act and interact to influence APB amongst surgical teams. We will develop new theory to advance understanding and identify knowledge gaps to inform further research.
\end{abstract}

Methods: The meta-ethnography will follow the seven-phase method described by Noblit and Hare. We will conduct a comprehensive search using eight databases (AMED, CINAHL, EMBASE, MEDLINE, MEDLINE-in-process, Web of Science, Cochrane Library and PsycINFO) with no date restrictions; forwards and backwards citation searches; and contacting first authors of relevant papers. Studies will be dual screened and included if they use recognised qualitative methods and analysis; focus on contextual factors associated with surgical APB within hospital settings; are available in full in English; and are relevant to the research question. Any disagreements between reviewers will be resolved through discussion to reach consensus. Included studies will be read repeatedly to illuminate key concepts and the relationship between key concepts across studies. Then, key concepts will be sorted into conceptual categories or 'piles' which will be further abstracted to form a conceptual framework explaining surgical APB. During the synthesis, emerging interpretations will be discussed with stakeholders (including authors of included studies where possible; surgical and stewardship practitioners; and patient representatives) to ensure new knowledge is meaningful.

Discussion: This research has several strengths: (1) the protocol has been written with reference to established guidance maximising rigour and transparency; (2) the multi-disciplinary research team bring varied interpretative repertoires and relevant methodological skills; and (3) stakeholders will be involved to ensure that findings are relevant, and disseminated via suitable channels, to support improved patient care.

Systematic review registration: PROSPERO CRD42020184343

Keywords: Antimicrobial decision-making, Antimicrobial prescribing behaviour, Meta-ethnography, Qualitative, Surgery, Synthesis

\footnotetext{
*Correspondence: hazel.parker1@nhs.net

'Pharmacy Department, Royal Devon and Exeter NHS Foundation Trust, Barrack Road, Exeter EX2 5DW, UK

Full list of author information is available at the end of the article
}

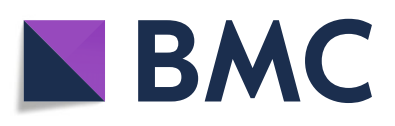

( ) The Author(s). 2020 Open Access This article is licensed under a Creative Commons Attribution 4.0 International License, which permits use, sharing, adaptation, distribution and reproduction in any medium or format, as long as you give appropriate credit to the original author(s) and the source, provide a link to the Creative Commons licence, and indicate if changes were made. The images or other third party material in this article are included in the article's Creative Commons licence, unless indicated otherwise in a credit line to the material. If material is not included in the article's Creative Commons licence and your intended use is not permitted by statutory regulation or exceeds the permitted use, you will need to obtain permission directly from the copyright holder. To view a copy of this licence, visit http://creativecommons.org/licenses/by/4.0/. The Creative Commons Public Domain Dedication waiver (http://creativecommons.org/publicdomain/zero/1.0/) applies to the data made available in this article, unless otherwise stated in a credit line to the data. 


\section{Background}

Antimicrobial resistance (AMR) represents a global patient safety risk. By 2050, AMR will be responsible for an estimated ten million deaths annually [1] unless policies are successfully implemented to tackle its spread. One of the key strategies to reduce AMR is antimicrobial stewardship which encompasses the careful and responsible use of antimicrobials to improve patient outcomes. Antimicrobial misuse-including unnecessary use; wrong dose, route or duration of therapy; wrong agent; delayed administration in critically ill patients and mistimed surgical prophylaxis-leads to increased AMR; hospitalacquired infections; and other antimicrobial-associated adverse events.

Twenty percent of human antimicrobial consumption occurs in hospitals, and over the last 5 years usage has increased by $6.3 \%$ (or $2.8 \%$ if adjusted for growth in patient admissions over this time) [2]. A high proportion of this prescribing occurs in surgical specialities where inappropriate prescribing has been widely reported [3-7] with numerous calls for action [8-11]. Around ten million patients undergo surgery within the National Health Service (NHS) each year [12] with advances in surgical technique and anaesthesia resulting in more patients, at increased risk of infection, being offered surgery [13]. A single dose of surgical antimicrobial prophylaxis (SAP) is vital for many procedures, usually on starting anaesthesia, to limit surgical site infection [13]. SAP efficacy relies on the availability of suitable agent(s) that 'cover' bacteria likely to be encountered during the surgical procedure. Antimicrobial treatment is only indicated if the patient develops an infection, for example, a surgical site infection. However, the prevalence of healthcare-associated infection is high amongst surgical patients (8.5\%), second only to intensive care, and $39.5 \%$ of surgical patients are prescribed an antimicrobial on any given day [14]. In English hospitals, 1 in 12 patients are administered SAP: about half receive more than the recommended single dose and a third receive more than $24 \mathrm{~h}$ of antimicrobial cover [14]. Antimicrobial treatment courses prescribed by surgeons are less likely to be compliant with evidence-based guidelines, compared to treatment courses prescribed in general medicine, and more likely to be escalated to broader spectrum agents (which have activity against a wide range of microorganisms) [15]. This is despite the wide availability of international [16], national [13, 17-20] and local [21] guidelines reinforcing the point that the provision of guidelines alone is insufficient to change practice [22]. There is an urgent need to improve surgical APB to minimise unintended patient harm.
Interventions that are informed by, and tailored to, a clear understanding of the contextual barriers to appropriate antimicrobial use are more likely to improve practice $[23,24]$. However, little attention has been paid to how interventions work in different contexts and for different prescribing groups [25] and many interventions do not use effective behaviour change techniques [26]. To bring about meaningful, sustained behaviour change, it is essential to understand the contextual factors influencing surgical APB. Then, holistic, contextsensitive interventions can be co-designed and delivered accordingly, to improve patient care.

Multiple reasons for antimicrobial misuse within surgery have been postulated, including a lack of training, experience or confidence; inadequate knowledge of local AMR epidemiology; misinterpretation of microbiology results; uncertain diagnosis and/or lack of guidance or institutional leadership [8]. Additionally, a growing body of qualitative studies have described surgical teams' APB, highlighting it as distinct from that of other physicians [27-29]. Surgeons view antimicrobial management as peripheral to their role. Fear of failure, risk of blame and lack of expertise all contribute to inappropriate antimicrobial use [27]. Professional hierarchies within and between specialities influence SAP decisions [30], with obstacles to timely administration including organisational communication, inconvenience, workflow, role perception and the low priority assigned to antimicrobials [31]. Post-operatively, the ward round becomes central to decision-making. However, rounds are often rushed owing to pressure on the surgeons to be in theatre; responsibility for antimicrobial prescribing is often delegated to junior staff; team members are rarely present for the entire round (reducing continuity); and juniors, usually responsible for keeping track of key decisions and tasks, are frequently sent away from the round to chase results, or are omitted from critical conversations. As a result, team members become unclear about which patients are prescribed antimicrobials [27].

A synthesis of qualitative studies on surgical APB is warranted to generate a comprehensive and transferrable theory that will inform future research and antimicrobial stewardship programmes [32]. Metaethnography is a well-established qualitative evidence synthesis methodology with origins in the interpretive paradigm. It was first developed by Noblit and Hare (1988) in the field of education, because their aggregative synthesis could not explain the failure of desegregation in schools, but it is now widely used in health and social care research [3335]. The seven-phase process integrates and compares findings from multiple qualitative studies facilitating the identification of overarching constructs 
and development of new theory [36]. Overarching practical knowledge in the form of a theory (system of ideas explaining phenomena) has the potential to support healthcare workers and policymakers by providing a complex and comprehensive conceptual understanding of things that cannot be 'pinned down' [37]. Our aim is to synthesise qualitative studies on surgical APB in hospital settings to explain how and why contextual factors act and interact to influence APB amongst surgical teams. We will develop new, clinically applicable, theory to advance understanding and identify knowledge gaps to inform further research.

\section{Methods}

The meta-ethnography has been registered with the International Prospective Register of Systematic Reviews (PROSPERO); registration number CRD42020184343. Protocol development was informed by recent advances in meta-ethnographic theory and practice $[33,34,36,38-42]$; and with reference to the Preferred Reporting Items for Systematic Review and Meta-analysis Protocols (PRIS MA-P) checklist (see Additional file). Data from eligible papers will be synthesised following the seven phases (see Fig. 1) outlined by Noblit and Hare; however, meta-ethnography is not a linear process and these phases will likely overlap and repeat as the synthesis proceeds [36]. Findings will be evaluated using the Confidence in Evidence from Reviews of Qualitative Research (CERQual) approach [43], and reported in accordance with the eMERGe reporting guidelines [44]. Ethical approval is not required for a synthesis of published peer-reviewed studies (http://www.hradecisiontools.org.uk/ethics/).

\section{Theoretical perspective}

We (HP, KM and NB) collaborated to prepare a $\mathrm{Na}$ tional Institute for Health Research funding application with the intention to build on previous work $[45,46]$ and this has now been funded. HP has worked as a National Health Service Hospital Pharmacist for the past 15 years, specialising in the field of antimicrobials since 2009, and is now a National Institute for Health Research (NIHR) Clinical Doctoral Research Fellow. JF and NB are Medical Sociologists with extensive qualitative research and meta-ethnography experience. KM is a Professor of Medical Education and has broad research experience including qualitative methods and analysis. SR is an experienced information specialist.

Meta-ethnography is an inductive, highly interpretive approach [36, 40]. We will be endeavouring to develop new interpretations from first-order data (primary study participant interpretations), and second-order data (author interpretations of participant interpretations) presented in the primary studies. To broaden perspective and to support the development of insightful, practical theory, we have formed a stakeholder group that will contribute to the synthesis. Stakeholders will include a consultant surgeon, anaesthetist and microbiologist; a hospital pharmacist; and several patient representatives. Additionally, technical support will be provided by an information specialist (SR) and a research fellow (EC) from the NIHR Applied Research Collaboration South West Peninsula Patient and Public Involvement team.

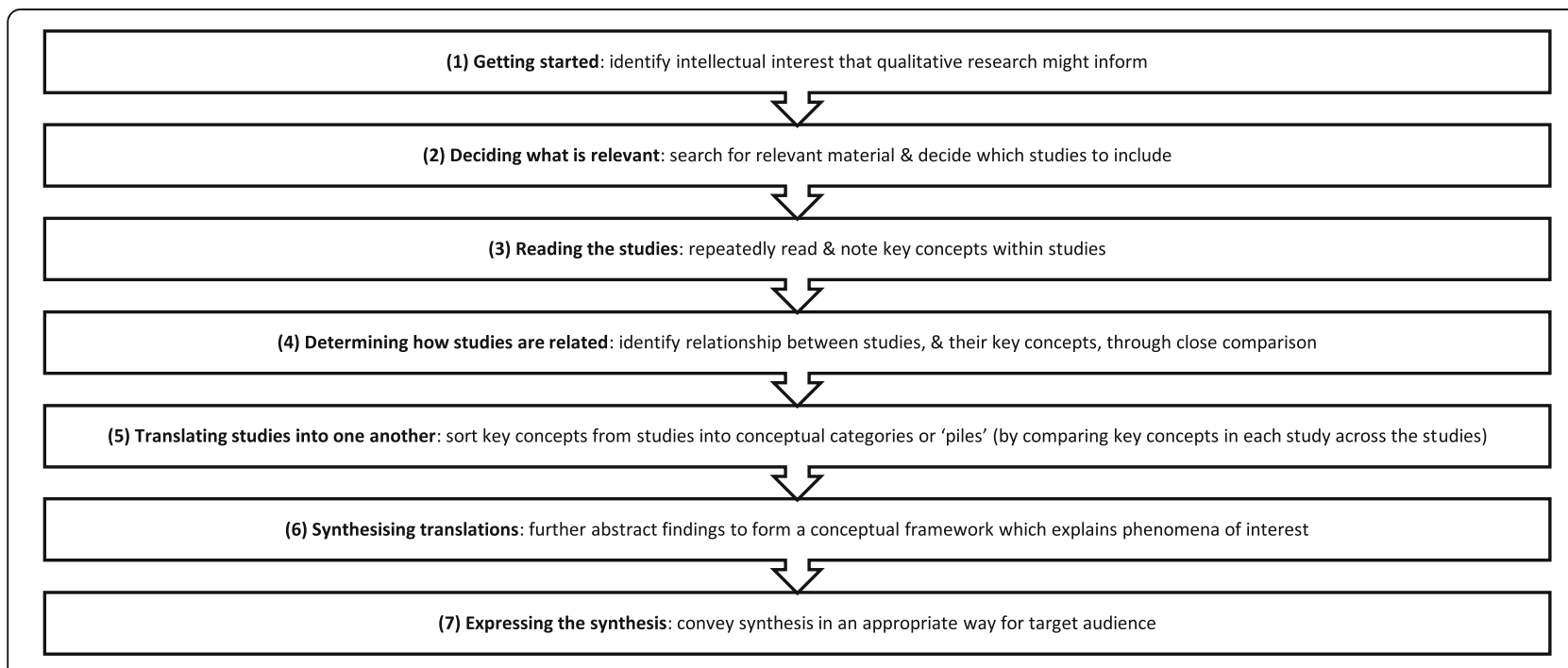

Fig. 1 Noblit and Hare's [36] seven phases of meta-ethnography 


\section{Phase one: getting started}

\section{Formulating the research question}

Our research question is how and why do contextual factors act and interact to influence surgical APB in hospital settings? Several qualitative studies have explored the subject. However, a synthesis offers us a tool to understand this body of work more fully, more deeply and more convincingly [47], whereas other approaches might remove context and/or impede explanation [36]. Meta-ethnography is the preferred qualitative evidence synthesis method as it is systematic and has the potential to preserve interpretive properties from the primary studies [48]. Furthermore, it aims to develop conceptual understanding [36], which aligns with our intentions, and has been implemented previously to develop theory about antimicrobial prescribing interventions in general practice [35]. As far as we know, this will be the first metaethnography addressing surgical APB.

\section{Phase two: deciding what is relevant Focus of the synthesis}

We decided to focus on studies that employed recognised qualitative methods and analysis (i.e. primary qualitative or mixed methods studies) to explore APB amongst surgical team members (from any surgical speciality). This is because conceptually rich studies are a pre-requisite for interpretive synthesis methods. Other types of studies (e.g. quantitative questionnaire studies with some open-ended free text questions) would be unlikely to provide the rich elaborations needed to make interpretations and to build understanding. Qualitative studies of APB in primary care, or those that did not differentiate surgical teams from non-surgical specialities in hospital settings, were not included because we view surgeons as a distinct group requiring a nuanced antimicrobial stewardship approach.

\section{Locating relevant studies}

$\mathrm{HP}$ and an information specialist (SR) will develop a search strategy (see Appendix), and will systematically search eight databases (AMED, CINAHL, EMBASE, MEDLINE, MEDLINE-in-process, Web of Science, Cochrane Library and PsycINFO) from their inception to identify potentially relevant studies. This combination of databases will enable representation from a range of domains including medical and allied health professional research and clinical practice; sociology; psychology and related disciplines. This is important as qualitative research is frequently catalogued outside the medical domain. The SPIDER tool [49] (see Table 1) has been used to provide structure for the search, although search terms will be individualised for each database. Because qualitative literature can be challenging to find [50], we will employ supplementary search methods [51] to identify additional suitable studies: (1) forwards and backwards citation searching using studies that meet the inclusion criteria for the meta-ethnography; and (2) we will contact experts in the field, including the authors of all included studies, to ask them to suggest any additional studies (including those in-press).

The purpose of the comprehensive search is to identify the relevant body of literature containing information on the contextual factors associated with surgical APB. A comprehensive approach has been chosen as (1) it will ensure that all relevant work is cited, to facilitate the development of theory and to prevent unhelpful research repetition/waste; and (2) it is more likely to resonate with our target audience (surgical and stewardship teams) who are more familiar with quantitative systematic reviews. Scoping searches suggest that the volume of applicable literature will be manageable. However, should the number of studies uncovered become unwieldy, we will use a purposive or theoretical sampling strategy in keeping with the epistemology of metaethnography [50].

\section{Inclusion and exclusion decisions}

All retrieved studies will be imported into Endnote reference management software and de-duplicated. HP and SR will then independently screen the studies based on the title and abstract.

Studies will be excluded if they do not have a qualitative component or do not describe APB in a surgical context (see Table 1). In the event of uncertainty or disagreement studies will be sought in full, in addition to the studies that definitely appear relevant, to be assessed by HP and one other author (KM or JF). Studies will be included if they use recognised qualitative methods (e.g. interviews, focus groups or observation) and analysis (e.g. framework analysis or thematic analysis); focus on contextual factors associated with surgical (any speciality) APB within a hospital setting, and are available in full in the English language. Any disagreement at the final screening stage will be resolved by consensus between three reviewers (HP, JF and $\mathrm{KM}$ ).

\section{Quality assessment}

No studies will be excluded based on quality alone [52]. However, all studies will be assessed using the qualitative Critical Appraisal Skills Programme (CASP) tool [53] to support careful and systematic reading [40] with consideration of a range of aspects [41]. Lower quality assessment scores-for example, due to poor reporting or abridged methods sections (often the case in medical journals)-does 
Table 1 SPIDER table of study inclusion and exclusion criteria

\begin{tabular}{|c|c|c|}
\hline & Inclusion criteria & Exclusion criteria \\
\hline Sample & $\begin{array}{l}\text { - Surgical teams (any members including surgeons, } \\
\text { trainee surgeons, anaesthetists, surgical nurses, } \\
\text { surgical pharmacists etc.) } \\
\text { - Secondary care setting including wards; out-patient } \\
\text { clinics; theatres etc. }\end{array}$ & $\begin{array}{l}\text { - Non-surgical specialities } \\
\text { - Other care settings, e.g. primary care; dentists } \\
\text { - Veterinary studies }\end{array}$ \\
\hline Phenomenon of interest & $\begin{array}{l}\text { - Antimicrobial/antibiotic prescribing behaviour } \\
\text { (treatment and/or prophylaxis) }\end{array}$ & - Prescribing behaviour related to other medication classes \\
\hline Design & $\begin{array}{l}\text { - Qualitative or mixed-method studies reporting } \\
\text { primary qualitative data collected using qualitative } \\
\text { methods (e.g. through direct observation; focus } \\
\text { groups or interviews) }\end{array}$ & $\begin{array}{l}\text { - Studies that report quantitative data only including } \\
\text { questionnaire studies with open-ended free text } \\
\text { questions }\end{array}$ \\
\hline Evaluation & $\begin{array}{l}\text { - Qualitative analysis of antimicrobial prescribing } \\
\text { behaviour (using any qualitative evaluation, e.g. } \\
\text { grounded theory; and framework analysis) }\end{array}$ & $\begin{array}{l}\text { - Studies that evaluate using quantitative methods only } \\
\text { - Studies that do not explicitly state the method of analysis }\end{array}$ \\
\hline Research type & $\begin{array}{l}\text { - Peer-reviewed journal articles } \\
\text { - Full text available } \\
\text { - English language }\end{array}$ & $\begin{array}{l}\text { - Reviews; protocols; theoretical work; editorials; opinion } \\
\text { pieces and grey literature } \\
\text { - Non-English language }\end{array}$ \\
\hline
\end{tabular}

not always reflect the quality of the research; however, it can draw the reviewer's attention to shortcomings in the interpretation of study findings that may have an impact on the results of the synthesis [38].

Three reviewers (HP, KM and JF) will then use a pragmatic approach, first described by Dixon-Woods et al. (2007) [54], to classify studies based on their perceived utility to the meta-ethnography (see Table 2). Those studies deemed 'irrelevant' or 'fatally flawed' will be excluded. Remaining papers-key papers; satisfactory papers; and questionable papers-will be included in the synthesis. Any disagreement regarding categorisation of a study will be resolved by consensus between the three reviewers. Additionally, synthesis messages derived from the included studies will be examined against 'key' papers (only) to test their contributions and promote further discussion and insight, consistent with previous work [41]. A Microsoft Excel spreadsheet will be used to collate study demographics; appraisal scores and inclusion/exclusion decisions.

\section{Phase three: reading the studies}

This stage of the meta-ethnography involves repeated careful reading of the studies to gain familiarity and to

Table 2 Study classifications [54]

\begin{tabular}{|c|c|}
\hline Category & Study characteristics \\
\hline Key papers & $\begin{array}{l}\text { Conceptually rich with the potential to make } \\
\text { an important contribution to the synthesis }\end{array}$ \\
\hline Satisfactory papers & Less valuable than key papers but still relevant \\
\hline Questionable papers & Uncertain contribution \\
\hline Irrelevant & Not relevant to the review question \\
\hline Fatally flawed & Study data not presented in a usable format \\
\hline
\end{tabular}

identify the main concepts described, i.e. what is each study telling us. Contextual information, such as study setting; participants (e.g. sub-speciality, grade and number included); research design and aim, will be recorded in a Microsoft Excel spreadsheet. Following repeated close reading ( $\mathrm{HP}, \mathrm{KM}$ and JF), the studies will be imported into the NVivo qualitative data analysis software. Key concepts (potentially explanatory ideas) from each study's results and/or discussion section will then be independently coded by two reviewers (HP and JF or KM). The coded data will include quotations from participants (first-order data) and quotations from the original study's authors (second-order data). Reviewers (HP, JF and KM) will then discuss and agree on the key concepts, recording them in a Microsoft Excel spreadsheet.

\section{Phase four: determining how the studies are related}

The next phase requires us to identify the relationship between the primary studies, and their key concepts, through a process of close comparison. We will begin to determine whether the synthesis is 'reciprocal' (primary studies' concepts are directly complementary), 'refutational' (primary studies' concepts oppose each other) or 'lines-of-argument'. In the latter case, primary studies identify different aspects of a larger phenomenon which when taken together offer a new interpretation; a 'whole' is discovered from a set of parts [36]. We are aware that metaethnographies frequently produce reciprocal or linesof-argument translations [55]. Lines of argument syntheses often bring together interrelated concepts, but may also represent a lack of attention to conflicting findings. We will actively seek disconfirming or contradictory findings and concepts. 
Phase five: translating the studies into one another Translation involves sorting the key concepts (from primary studies) into conceptual categories or 'piles' [42]. $\mathrm{HP}, \mathrm{KM}$ and JF will independently compare key concepts across the primary studies, grouping them all into conceptual categories (third-order data) with a definition of what each conceptual category encompasses. The conceptual categories will be developed inductively, through a process of constant comparison (of key concepts), rather than according to any a priori theory, although we recognise each reviewer's interpretations will be influenced by their backgrounds. The reviewers will then compare interpretations and, with input from stakeholders, collaboratively develop a final list of conceptual categories, which will be tested against the primary studies to ensure a good fit. The multi-disciplinary input will enable us to challenge our own understandings and will support the identification of a range of possible analytic interpretations.

\section{Phase six: synthesising translations}

Synthesising translations is the on-going process whereby findings are further abstracted to form a conceptual framework [42], which explains the phenomena of interest. It cannot be reduced to a set of mechanistic tasks [39] but will involve three reviewers (HP, KM and JF) working collaboratively to 'make sense' of the conceptual categories, with the aim of developing a new theory that explains how and why contextual factors act and interact to influence APB amongst surgical teams. If appropriate, a visual way of representing the findings will be developed iteratively, to convey the theory.

Emerging interpretations will be discussed with (1) the authors of the primary studies (where possible) to test the validity of our third-order interpretations; (2) academic and surgical/stewardship audiences to receive feedback, for example at departmental seminars, conferences and methodological discussion fora and (3) our wider stakeholder group to ensure that the knowledge is applicable and meaningful. Additionally, we will assess the synthesis findings using the CERQual approach [43] to transparently determine how much confidence can be placed in them.

\section{Phase seven: expressing the synthesis}

Findings from the meta-ethnography will be published in a peer-reviewed journal; presented at suitable fora (including surgical and infection conferences and relevant teaching) and made available to patients and members of the public. We will work with key stakeholders and patient representatives to ensure that the media are acceptable to their target audience and disseminated via the most effective channels.

\section{Discussion}

As far as we know, there is no qualitative synthesis that explains how and why contextual factors interact to influence surgical APB. This knowledge is key to the development and implementation of effective, sustainable, interventions to improve practice. The meta-ethnography will develop new theory to broaden understanding of how and why contextual factors act and interact to influence APB amongst surgical teams. Insights will highlight research gaps and inform the development of context-fit quality-improvement interventions to change practice and to improve patient outcomes.

There are several strengths to the research: (1) the protocol has been written with reference to established guidance maximising rigour and transparency; (2) the multidisciplinary research team brings varied interpretative repertoires and deep experiential knowledge of a wide range of qualitative methods, including qualitative synthesis, which is key in developing theory and explanation $[47,55]$ and (3) key stakeholders, including patient representatives, will continue to be included throughout the research process to ensure that findings are relevant to healthcare workers and patients who undergo surgery. Furthermore, the involvement of this broad group of stakeholders will support us in disseminating findings via suitable channels to facilitate improvement in practice, for example, one of our stakeholder group is a consultant surgeon and clinical lead for the South West Patient Safety Collaborative.

\section{Appendix \\ MEDLINE search strategy}

\begin{tabular}{|c|c|}
\hline No. & Search \\
\hline 1 & $\begin{array}{l}\text { ((surgery or surgical or theatre or operat*) adj3 (nurse* or nursing } \\
\text { or doctor } \text { or pharmacist* or trainee* or registrar* or junior* or }^{*} \text { or }^{*} \text { medic }^{*} \text { or physician* or consultant* or team* or group* or } \\
\text { profession*)).tw. }\end{array}$ \\
\hline 2 & (surgeon* or anaesthetist* or anesthetist*).tw. \\
\hline 3 & exp Surgeons/ \\
\hline 4 & exp Anesthetists/ \\
\hline 5 & Perioperative Nursing/ \\
\hline 6 & Pharmacists/ \\
\hline 7 & or/1-6 \\
\hline 8 & $\begin{array}{l}\text { (prescrib* or prescrip* or "decision making" or administer or } \\
\text { administration).tw. }\end{array}$ \\
\hline 9 & $\begin{array}{l}\text { ((drug* or medicine* or medication*) adj2 (administ* or utili?ation } \\
\left.\text { or error* or use }{ }^{*}\right) \text {.tw. }\end{array}$ \\
\hline 10 & exp Prescriptions/ \\
\hline 11 & exp Drug Utilization/ \\
\hline 12 & Inappropriate Prescribing/ \\
\hline 13 & Prescription Drugs/ \\
\hline 14 & Drug Prescriptions/ \\
\hline 15 & Medication Errors/ \\
\hline 16 & exp Decision Making/ \\
\hline 17 & or/8-16 \\
\hline 18 & (antimicrobial* or "anti microbial*" or antibiotic" or "anti bi \\
\hline
\end{tabular}




\section{Appendix (Continued)}

\begin{tabular}{|c|c|}
\hline No. & Search \\
\hline & 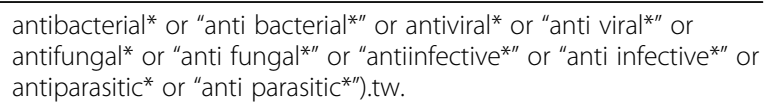 \\
\hline 19 & Anti-Infective Agents/ \\
\hline 20 & exp Anti-Bacterial Agents/ \\
\hline 21 & exp Antifungal Agents/ \\
\hline 22 & exp Anti-Infective Agents, Urinary/ \\
\hline 23 & exp Antiparasitic Agents/ \\
\hline 24 & exp Antiviral Agents/ \\
\hline 25 & Antibiotic Prophylaxis/ \\
\hline 26 & Post-Exposure Prophylaxis/ \\
\hline 27 & Pre-Exposure Prophylaxis/ \\
\hline 28 & or/18-27 \\
\hline 29 & 7 and 17 and 28 \\
\hline 30 & interview:.mp. \\
\hline 31 & experience:mp. \\
\hline 32 & qualitative:.tw. \\
\hline 33 & 30 or 31 or 32 \\
\hline 34 & 29 and 33 \\
\hline
\end{tabular}

\section{Supplementary information}

Supplementary information accompanies this paper at https://doi.org/10. 1186/s13643-020-01477-5.

Additional file 1. PRISMA-P 2015 Checklist

\section{Abbreviations}

APB: Antimicrobial prescribing behaviour; AMR: Antimicrobial resistance; CASP: Critical Appraisal Skills Programme; NIHR: National Institute for Health Research; SAP: Surgical antimicrobial prophylaxis

\section{Acknowledgements}

We would like to gratefully acknowledge the important contributions of the NIHR for funding the research; Dr. Emma Cockcroft who has helped plan the integration of patient and public involvement (PPI) throughout the research; Dr. Esmita Charani who has provided advice and mentoring (to HP); key stakeholders: Mr. Rob Bethune, Dr. Harry Pugh, Dr. Alexis Taylor, Mrs. Ali Hodgetts, Dr. Kieran Hand and Dr. Robert Porter who contributed time and ideas to support the research and all PPI persons, academics and healthcare workers who have given us feedback.

\section{Authors' contributions}

$\mathrm{HP}, \mathrm{NB}$ and $\mathrm{KM}$ were responsible for conceiving the review. HP, NB, JF and KM designed the protocol iteratively. SR advised on the choice of search methods and worked with HP to develop the MEDLINE search strategy. HP was responsible for preparing successive drafts of the manuscript. All authors approved the final version of the protocol for publication. HP is the guarantor of the review.

\section{Authors' information}

HP is a National Health Service hospital antimicrobial pharmacist and National Institute for Health Research (NIHR) clinical doctoral research fellow. JF is a Medical Sociologist and experienced nurse. She undertakes research about health service delivery and patient experience using qualitative and mixed methods and has completed several meta-ethnographies.
NB is a Professor of Applied Health Care Research and is an experienced qualitative researcher. She contributed to the adoption of meta-ethnography in healthcare research and has completed several meta-ethnographies. $\mathrm{KM}$ is a Professor of Medical Education. She researches issues that impact doctors-in-training using wide-ranging methodologies including systematic and realist literature reviews; quantitative, qualitative and mixed methods. SR is an Information Specialist. She works in the Peninsular Technology Assessment Group (PenTAG), at the University of Exeter, designing and running systematic literature searches for a variety of projects including technology appraisals.

\section{Funding}

This report is an independent research supported by Health Education England and the National Institute for Health Research (HEE/NIHR ICA Programme Clinical Doctoral Research Fellowship, Miss Hazel Parker, ICACDRF-2018-04-ST2-019). The views expressed in this publication are those of the author(s) and not necessarily those of the NHS, the National Institute for Health Research or the Department of Health and Social Care. The funder, sponsor and authors' institutions have had no role in developing the protocol.

\section{Availability of data and materials}

Not applicable.

Ethics approval and consent to participate

Not applicable.

\section{Consent for publication}

Not applicable.

\section{Competing interests}

The authors declare that they have no competing interests.

\section{Author details}

${ }^{1}$ Pharmacy Department, Royal Devon and Exeter NHS Foundation Trust, Barrack Road, Exeter EX2 5DW, UK. ${ }^{2}$ South Cloisters, University of Exeter, St Luke's Campus, Heavitree Road, Exeter EX1 2LU, UK.

Received: 30 June 2020 Accepted: 8 September 2020

Published online: 10 October 2020

\section{References}

1. O'Neill J: Tackling drug-resistant infections globally: final report and recommendations. In.: 2016.

2. Public Health England: English surveillance programme for antimicrobial utilisation and resistance (ESPAUR) report 2018-2019. In.

3. Charani E, Ahmad R, Tarrant C, Birgand G, Leather A, Mendelson M, Moonesinghe SR, Sevdalis N, Singh S, Holmes A. Opportunities for system level improvement in antibiotic use across the surgical pathway. Int J Infectious Diseases. 2017:60:29-34.

4. Goede WJ, Lovely JK, Thompson RL, Cima RR. Assessment of prophylactic antibiotic use in patients with surgical site infections. Hosp Pharm. 2013:48(7):560-7.

5. Hohmann C, Eickhoff C, Radziwill R, Schulz M. Adherence to guidelines for antibiotic prophylaxis in surgery patients in German hospitals: a multicentre evaluation involving pharmacy interns. Infection. 2012;40(2):131-7.

6. Gorecki P, Schein M, Rucinski JC, Wise L. Antibiotic administration in patients undergoing common surgical procedures in a community teaching hospital: the chaos continues. World J Surgery. 1999;23(5):429-33.

7. Tourmousoglou CE, Yiannakopoulou EC, Kalapothaki V, Bramis J, Papadopoulos JS. Adherence to guidelines for antibiotic prophylaxis in general surgery: a critical appraisal. J Antimicrobial Chemotherapy. 2007;61(1):214-8.

8. Cakmakci M. Antibiotic stewardship programmes and the surgeon's role. J Hosp Infect. 2015;89(4):264-6.

9. Global Alliance for Infection in Surgery Working Group. A global declaration on appropriate use of antimicrobial agents across the surgical pathway. Surg Infect (Larchmt). 2017;18(8):846-53.

10. Goff DA, File TM. The risk of prescribing antibiotics "just-in-case" there is infection. Seminars in Colon and Rectal Surgery. 2018;29(1):44-8.

11. Sartelli M, Duane TM, Catena F, Tessier JM, Coccolini F, Kao LS, De Simone B, Labricciosa FM, May AK, Ansaloni L, et al. Antimicrobial stewardship: a call to action for surgeons. Surg Infect (Larchmt). 2016;17(6):625-31. 
12. Perioperative medicine the pathway to better surgical care [https://www. rcoa.ac.uk/media/4726 [accessed 29.05.20]].

13. NICE: Surgical site infections: prevention and treatment NICE guideline [NG125]. 2019.

14. Public Health England: English surveillance programme for antimicrobial utilisation and resistance (ESPAUR) Report 2017. In.

15. Charani E, de Barra E, Rawson TM, Gill D, Gilchrist M, Naylor NR, Holmes AH. Antibiotic prescribing in general medical and surgical specialties: a prospective cohort study. Antimicrob Resist Infect Control. 2019:8:151.

16. World Health Organisation: Global guidelines for the prevention of surgical site infection. In.; 2016: 184

17. Berríos-Torres SI, Umscheid CA, Bratzler DW, Leas B, Stone EC, Kelz RR, Reinke CE, Morgan S, Solomkin JS, Mazuski JE, et al. Centers for disease control and prevention guideline for the prevention of surgical site infection, 2017. JAMA Surgery. 2017;152(8):784

18. Bratzler DW, Dellinger EP, Olsen KM, Perl TM, Auwaerter PG, Bolon MK, Fish DN, Napolitano LM, Sawyer RG, Slain D, et al. Clinical practice guidelines for antimicrobial prophylaxis in surgery. American Journal of Health-System Pharmacy. 2013;70(3):195-283.

19. Solomkin JS, Mazuski JE, Bradley JS, Rodvold KA, Goldstein EJ, Baron EJ, O'Neill PJ, Chow AW, Dellinger EP, Eachempati SR, et al. Diagnosis and management of complicated intra-abdominal infection in adults and children: guidelines by the Surgical Infection Society and the Infectious Diseases Society of America. Clin Infect Dis. 2010;50(2):133-64.

20. SIGN: SIGN 104: antibiotic prophylaxis in surgery. 2014.

21. Wickens HJ, Farrell S, Ashiru-Oredope DAl, Jacklin A, Holmes A, Cooke J, Sharland M, Ashiru-Oredope D, McNulty C, Dryden M, et al. The increasing role of pharmacists in antimicrobial stewardship in English hospitals. J Antimicrobial Chemotherapy. 2013;68(11):2675-81.

22. Cabana MD, Rand CS, Powe NR, Wu AW, Wilson MH, Abboud PA, Rubin HR. Why don't physicians follow clinical practice guidelines? A framework for improvement. JAMA. 1999;282(15):1458-65.

23. Baker R, Camosso-Stefinovic J, Gillies C, Shaw EJ, Cheater F, Flottorp S, Robertson $\mathrm{N}$, Wensing M, Fiander M, Eccles MP, et al. Tailored interventions to address determinants of practice. Cochrane Database Syst Rev. 2015;4:CD005470.

24. Papoutsi C, Mattick K, Pearson M, Brennan N, Briscoe S, Wong G. Social and professional influences on antimicrobial prescribing for doctors-in-training: a realist review. Journal of Antimicrobial Chemotherapy. 2017;72(9):2418-30.

25. Brennan N, Mattick K: A systematic review of educational interventions to change behaviour of prescribers in hospital settings, with a particular emphasis on new prescribers. 2013, 75(2):359-372.

26. Davey P, Marwick CA, Scott CL, Charani E, McNeil K, Brown E, Gould IM, Ramsay CR, Michie $S$. Interventions to improve antibiotic prescribing practices for hospital inpatients. Cochrane Database Syst Rev. 2017;2:CD003543.

27. Charani E, Tarrant C, Moorthy K, Sevdalis N, Brennan L, Holmes AH. Understanding antibiotic decision making in surgery-a qualitative analysis. Clin Microbiol Infect. 2017;23(10):752-60.

28. Charani E, Ahmad R, Rawson TM, Castro-Sanchez E, Tarrant C, Holmes AH. The differences in antibiotic decision-making between acute surgical and acute medical teams: an ethnographic study of culture and team dynamics. Clin Infect Dis. 2019;69(1):12-20.

29. Broom J, Broom A, Kirby E, Post JJ. Improvisation versus quideline concordance in surgical antibiotic prophylaxis: a qualitative study. Infection. 2018:46(4):541-8.

30. Broom JK, Broom AF, Kirby ER, Post JJ. How do professional relationships influence surgical antibiotic prophylaxis decision making? A qualitative study. Am J Infect Control. 2018;46(3):311-5.

31. Tan JA, Naik VN, Lingard L. Exploring obstacles to proper timing of prophylactic antibiotics for surgical site infections. Qual Saf Health Care. 2006;15(1):32-8.

32. Rzewuska M, Charani E, Clarkson JE, Davey PG, Duncan EM, Francis JJ, Gillies K, Kern WV, Lorencatto F, Marwick CA, et al. Prioritizing research areas for antibiotic stewardship programmes in hospitals: a behavioural perspective consensus paper. Clin Microbiol Infect. 2019;25(2):163-8.

33. France EF, Ring N, Thomas R, Noyes J, Maxwell M, Jepson R. A methodological systematic review of what's wrong with meta-ethnography reporting. BMC Med Res Methodol. 2014;14:119.

34. France EF, Uny I, Ring N, Turley RL, Maxwell M, Duncan EAS, Jepson RG, Roberts $\mathrm{R}$, Noyes J. A methodological systematic review of meta-ethnography conduct to articulate the complex analytical phases. BMC Med Res Methodol. 2019;19(1):35.

35. Germeni E, Frost J, Garside R, Rogers M, Valderas JM, Britten N. Antibiotic prescribing for acute respiratory tract infections in primary care: an updated and expanded meta-ethnography. Br J Gen Pract. 2018;68(674):e633-45.
36. Noblit GW. and Hare RD.: Meta-ethnography: synthesizing qualitative studies, vol. Qualitative research methods series 11; 1988.

37. Reeves $\mathrm{S}$, Albert M, Kuper A, Hodges BD. Why use theories in qualitative research? BMJ. 2008:337:a949.

38. Atkins S, Lewin S, Smith H, Engel M, Fretheim A, Volmink J. Conducting a meta-ethnography of qualitative literature: lessons learnt. BMC Med Res Methodol. 2008;8:21.

39. Britten N, Campbell R, Pope C, Donovan J, Morgan M, Pill R. Using meta ethnography to synthesise qualitative research: a worked example. J Health Serv Res Policy. 2002;7(4):209-15.

40. Campbell R, Pound P, Morgan M, Daker-White G, Britten N, Pill R, Yardley L, Pope C, Donovan J. Evaluating meta-ethnography: systematic analysis and synthesis of qualitative research. Health Technol Assess. 2011;15(43):1-164.

41. Malpass A, Shaw A, Sharp D, Walter F, Feder G, Ridd M, Kessler D: : "Medication career" or "moral career"? The two sides of managing antidepressants: a meta-ethnography of patients' experience of antidepressants. Soc Sci Med 2009, 68(1):154-168.

42. Toye F, Seers K, Allcock N, Briggs M, Carr E, Barker K. Meta-ethnography 25 years on: challenges and insights for synthesising a large number of qualitative studies. BMC Med Res Methodol. 2014;14:80.

43. Lewin S, Glenton C, Munthe-Kaas H, Carlsen B, Colvin CJ, Gülmezoglu M, Noyes J, Booth A, Garside R, Rashidian A. Using qualitative evidence in decision making for health and social interventions: an approach to assess confidence in findings from qualitative evidence syntheses (GRADECERQual). PLOS Medicine. 2015;12(10):e1001895.

44. France EF, Cunningham M, Ring N, Uny I, Duncan EAS, Jepson RG, Maxwell M, Roberts RJ, Turley RL, Booth A, et al. Improving reporting of metaethnography: the eMERGe reporting guidance. BMC Medical Research Methodology. 2019:19(1)

45. Parker HM, Mattick K. The determinants of antimicrobial prescribing among hospital doctors in England: a framework to inform tailored stewardship interventions. Br J Clin Pharmacol. 2016;82(2):431-40.

46. Kajamaa A, Mattick K, Parker H, Hilli A, Rees C. Trainee doctors' experiences of common problems in the antibiotic prescribing process: an activity theory analysis of narrative data from UK hospitals. BMJ Open. 2019;9(6): e028733.

47. Thorne S. Metasynthetic madness: what kind of monster have we created? Qual Health Res. 2017;27(1):3-12.

48. Noyes J \& Lewin S. Chapter 6: Supplemental guidance on selecting a method of qualitative evidence synthesis alQEwCIRINJ, Booth A, Hannes K, Harden A, Harris J, Lewin S, Lockwood C (editors), Supplementary Guidance for Inclusion of Qualitative Research in Cochrane Systematic Reviews of Interventions. Version 1 (updated August 2011). Cochrane CollabQualitative Methods Group, 2011. Available from: http://carmg.cochrane.org/ supplemental-handbook-guidance [accessed 26.04.2020].

49. Cooke A, Smith D, Booth A. Beyond PICO: the SPIDER tool for qualitative evidence synthesis. Qual Health Res. 2012;22(10):1435-43.

50. Booth A. Searching for qualitative research for inclusion in systematic reviews: a structured methodological review. Syst Rev. 2016;5:74.

51. Cooper C, Booth A, Britten N, Garside R. A comparison of results of empirical studies of supplementary search techniques and recommendations in review methodology handbooks: a methodological review. Syst Rev. 2017;6(1):234.

52. Grant MJ, Booth A. A typology of reviews: an analysis of 14 review types and associated methodologies. Health Info Libraries J. 2009;26(2):91-108.

53. Critical Appraisal Skills Programme: CASP Qualitative Checklist. [On-line] 2018

54. Dixon-Woods M, Sutton A, Shaw R, Miller T, Smith J, Young B, Bonas S, Booth $A$, Jones $D$. Appraising qualitative research for inclusion in systematic reviews: a quantitative and qualitative comparison of three methods. J Health Serv Res Policy. 2007;12(1):42-7.

55. Britten N, Garside R, Pope C, Frost J, Cooper C. Asking more of qualitative synthesis: a response to Sally Thorne. Qual Health Res. 2017;27(9):1370-6.

\section{Publisher's Note}

Springer Nature remains neutral with regard to jurisdictional claims in published maps and institutional affiliations. 